\title{
Editorial
}

\section{H.E.S.S. phase-I observations of the plane of the Milky Way}

Of the three currently operating large Imaging Atmospheric Cherenkov Telescopes (IACT), the Namibia-based High Energy Stereoscopic System (H.E.S.S.) has the best access to the inner Galactic plane. Devoting 2700 hours to a survey of the Galactic plane, the H.E.S.S. Collaboration has covered the $l=250 \mathrm{deg}$ to $65 \mathrm{deg}$ longitude range for latitudes $|b|<3 \mathrm{deg}$, with 5 arcmin angular resolution.

In this issue, we publish a series of papers that presents the observations, analyzes many of the 78 detected compact sources, and makes the sky maps available in FITS format. By covering a wide range of objects, from pulsar wind nebulae to gamma-ray binaries through supernova remnants, these papers illustrate the great potential of IACTs to study the most energetic phenomena in the Galaxy and what can be expected from the planned multinational Cherenkov Telescope Array (CTA).

Thierry Forveille, Sergio Campana, and Steve Shore Astronomy \& Astrophysics Editors 